\title{
Discovery of a warm, dusty giant planet around HIP $65426^{\star, \star \star}$
}

G. Chauvin ${ }^{1,2}$, S. Desidera ${ }^{3}$, A.-M. Lagrange ${ }^{1}$, A. Vigan $^{4}$, R. Gratton ${ }^{3}$, M. Langlois ${ }^{4,5}$, M. Bonnefoy ${ }^{1}$, J.-L. Beuzit ${ }^{1}$, M. Feldt ${ }^{6}$, D. Mouillet ${ }^{1}$, M. Meyer ${ }^{7,8}$, A. Cheetham ${ }^{9}$, B. Biller ${ }^{6,10}$, A. Boccaletti $^{11}$, V. D'Orazi ${ }^{3}$, R. Galicher $^{11}$, J. Hagelberg ${ }^{1}$, A.-L. Maire ${ }^{6}$, D. Mesa ${ }^{3}$, J. Olofsson ${ }^{6,12}$, M. Samland ${ }^{6}$, T. O. B. Schmidt ${ }^{11}$, E. Sissa ${ }^{3}$, M. Bonavita ${ }^{3,10}$, B. Charnay ${ }^{11}$, M. Cudel $^{1}$, S. Daemgen ${ }^{7}$, P. Delorme ${ }^{1}$, P. Janin-Potiron ${ }^{13}$, M. Janson ${ }^{6,14}$, M. Keppler ${ }^{6}$, H. Le Coroller ${ }^{4}$,

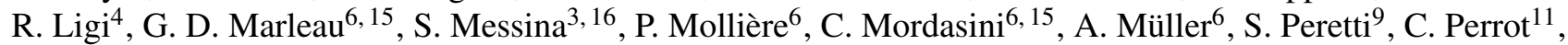
L. Rodet ${ }^{1}$, D. Rouan ${ }^{11}$, A. Zurlo ${ }^{3}{ }^{17}$, C. Dominik ${ }^{18}$, T. Henning ${ }^{6}$, F. Menard ${ }^{1}$, H.-M. Schmid ${ }^{7}$, M. Turatto ${ }^{3}$, S. Udry ${ }^{9}$, F. Vakili1 ${ }^{13}$, L. Abe ${ }^{13}$, J. Antichi ${ }^{19}$, A. Baruffolo ${ }^{3}$, P. Baudoz ${ }^{11}$, J. Baudrand ${ }^{11}$, P. Blanchard ${ }^{4}$, A. Bazzon ${ }^{7}$, T. Buey ${ }^{11}$, M. Carbillet ${ }^{13}$, M. Carle ${ }^{4}$, J. Charton ${ }^{1}$, E. Cascone ${ }^{20}$, R. Claudi ${ }^{3}$, A. Costille ${ }^{4}$, A. Deboulbe ${ }^{1}$, V. De Caprio ${ }^{20}$, K. Dohlen ${ }^{4}$, D. Fantinel ${ }^{3}$, P. Feautrier ${ }^{1}$, T. Fusco ${ }^{21}$, P. Gigan ${ }^{11}$, E. Giro ${ }^{3}$, D. Gisler ${ }^{7}$, L. Gluck ${ }^{1}$, N. Hubin ${ }^{22}$, E. Hugot ${ }^{4}$, M. Jaquet ${ }^{4}$, M. Kasper ${ }^{22}$, F. Madec ${ }^{4}$, Y. Magnard ${ }^{1}$, P. Martinez ${ }^{13}$, D. Maurel ${ }^{1}$, D. Le Mignant ${ }^{4}$, O. Möller-Nilsson ${ }^{6}$, M. Llored ${ }^{4}$, T. Moulin ${ }^{1}$, A. Origné ${ }^{4}$, A. Pavlov ${ }^{6}$, D. Perret ${ }^{11}$, C. Petit ${ }^{21}$, J. Pragt ${ }^{23}$, P. Puget ${ }^{1}$, P. Rabou ${ }^{1}$, J. Ramos ${ }^{6}$, R. Rigal ${ }^{18}$, S. Rochat ${ }^{1}$, R. Roelfsema ${ }^{23}$, G. Rousset ${ }^{11}$, A. Roux ${ }^{1}$, B. Salasnich ${ }^{3}$, J.-F. Sauvage ${ }^{21}$, A. Sevin ${ }^{11}$, C. Soenke ${ }^{22}$, E. Stadler ${ }^{1}$, M. Suarez ${ }^{19}$, L. Weber ${ }^{9}$, F. Wildi ${ }^{9}$, S. Antoniucci ${ }^{24}$, J.-C. Augereau ${ }^{1}$, J.-L. Baudino ${ }^{11,25}$,

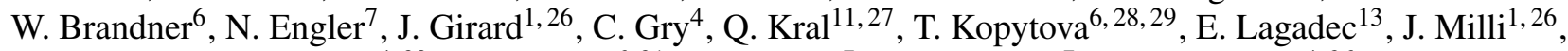
C. Moutou ${ }^{4,30}$, J. Schlieder ${ }^{6,31}$, J. Szulágyi ${ }^{7}$, C. Thalmann ${ }^{7}$, and Z. Wahhaj ${ }^{4,26}$

(Affiliations can be found after the references)

Received 10 May 2017 / Accepted 13 June 2017

\section{ABSTRACT}

Aims. The SHINE program is a high-contrast near-infrared survey of 600 young, nearby stars aimed at searching for and characterizing new planetary systems using VLT/SPHERE's unprecedented high-contrast and high-angular-resolution imaging capabilities. It is also intended to place statistical constraints on the rate, mass and orbital distributions of the giant planet population at large orbits as a function of the stellar host mass and age to test planet-formation theories.

Methods. We used the IRDIS dual-band imager and the IFS integral field spectrograph of SPHERE to acquire high-contrast coronagraphic differential near-infrared images and spectra of the young A2 star HIP 65426. It is a member of the 17 Myr old Lower Centaurus-Crux association. Results. At a separation of 830 mas (92 au projected) from the star, we detect a faint red companion. Multi-epoch observations confirm that it shares common proper motion with HIP 65426. Spectro-photometric measurements extracted with IFS and IRDIS between 0.95 and $2.2 \mu \mathrm{m}$ indicate a warm, dusty atmosphere characteristic of young low-surface-gravity L5-L7 dwarfs. Hot-start evolutionary models predict a luminosity consistent with a 6-12 $M_{\mathrm{Jup}}, T_{\mathrm{eff}}=1300-1600 \mathrm{~K}$ and $R=1.5 \pm 0.1 R_{\mathrm{Jup}}$ giant planet. Finally, the comparison with Exo-REM and PHOENIX BT-Settl synthetic atmosphere models gives consistent effective temperatures but with slightly higher surface gravity solutions of $\log (g)=4.0-5.0$ with smaller radii $\left(1.0-1.3 R_{\text {Jup }}\right)$.

Conclusions. Given its physical and spectral properties, HIP $65426 \mathrm{~b}$ occupies a rather unique placement in terms of age, mass, and spectral-type among the currently known imaged planets. It represents a particularly interesting case to study the presence of clouds as a function of particle size, composition, and location in the atmosphere, to search for signatures of non-equilibrium chemistry, and finally to test the theory of planet formation and evolution.

Key words. instrumentation: adaptive optics - instrumentation: high angular resolution - methods: observational planets and satellites: atmospheres - planets and satellites: formation

\section{Introduction}

More than a decade of direct imaging surveys targeting several hundred young, nearby stars have revealed that the occurrence of giant planets at wide orbits $(\geq 20-40 \mathrm{au})$ is relatively low (e.g. Bowler 2016). Despite the relatively small number of discoveries compared with other techniques, such as radial velocity and transit, each new imaged giant planet has provided

\footnotetext{
* Based on observations collected at La Silla and Paranal Observatory, ESO (Chile) Program ID: 097.C-0865 and 098.C-0209 (SPHERE). $\star \star$ The planet spectrum is only available at the CDS via anonymous ftp to cdsarc.u-strasbg. fr $(130.79 .128 .5)$ or via http://cdsarc.u-strasbg.fr/viz-bin/qcat?J/A+A/605/L9
}

unique clues on the formation, evolution and physics of young Jupiters. The latest generation of planet imagers, SPHERE (Beuzit et al. 2008), GPI (Macintosh et al. 2014) and SCExAO (Jovanovic et al. 2016), now combine innovative extreme adaptive optics systems with coronagraphic and differential imaging techniques. They offer unprecedented detection, astrometric and spectrophotometric capabilities which allow us to discover and characterize fainter and closer giant planets, such as the recent discovery of 51 Erib ( $2 M_{\text {Jup }}$ at 14 au, T5-type, of age 20 Myr; Macintosh et al. 2015; Samland et al. 2017). The SHINE (SpHere INfrared survey for Exoplanets) survey is currently surveying 600 young, nearby stars as part of the SPHERE Guaranteed Time Observations. In this survey, we observed 
the close environment of the young, star HIP 65426. The deep coronographic near-infrared observations revealed the presence of a young, warm, and dusty L5-L7 massive jovian planet, hereafter HIP 65426 b, located at about 92 au (projected distance). We describe below the observing set-up and data reduction, the physical properties of HIP $65426 \mathrm{~b}$, and finally discuss this new discovery in comparison to other imaged planetary systems and current planet formation and evolution theories.

\section{Host star properties}

HIP 65426 is an A2-type $(H=6.853 \pm 0.049$ mag, Cutri et al. 2003; $d=111.4 \pm 3.8 \mathrm{pc}$, Gaia Collaboration 2016) member of the Lower Centaurus-Crux (hereafter LCC) association (de Zeeuw et al. 1999; Rizzuto et al. 2011). A detailed summary of the main stellar properties as found in the literature is given in Appendix A. To refine them, the star was observed with HARPS (Mayor et al. 2003) on January 16th, 17th and 18th, $2017^{1}$. We measured the stellar absolute radial and projected rotational velocities using a custom cross-correlation function procedure specifically tailored for fast-rotating early-type stars. Values of $V_{\text {rad }}=5.2 \pm 1.3 \mathrm{~km} \mathrm{~s}^{-1}$ and $v \sin i=299 \pm 9 \mathrm{~km} \mathrm{~s}^{-1}$ were found (Appendix B). A marginally significant radial velocity difference was found between the three epochs. This is probably due to stellar pulsations as suggested by the periodicity $(P \sim 0.135$ days $)$ found from the HIPPARCos photometric time series. HIP 65426 is one of the fastest rotators known with similar spectral type (Zorec \& Royer 2012) and is therefore likely viewed along mid- to high-inclinations with respect to the rotation axis. Given its spectral type, the observed colors of HIP 65426 suggest a small value of reddening consistent with estimates reported in Chen et al. (2012). Assuming a metallicity for the LCC that is close to solar (Viana Almeida et al. 2009), theoretical isochrones predict an age of $14 \pm 4 \mathrm{Myr}$ for LCC members in the vicinity of HIP 65426 (we refer to Appendix A for details). SPHERE and HARPS observations do not show evidence of binarity (Appendix C). Finally, according to Chen et al. (2012), no IR excess is reported for this star. Our own SED analysis confirms this finding with only a tentative marginal excess at WISE W4 (Appendix D).

\section{Observations and data reduction}

HIP 65426 was observed on May 30th, 2016 under unstable conditions (strong wind) with SPHERE. The observations were then repeated on June 26th, 2016. The data were acquired in IRDIFS pupil-tracking mode with the 185 mas diameter apodized-Lyot coronograph (Carbillet et al. 2011; Guerri et al. 2011), using IRDIS (Dohlen et al. 2008) in dual-band imaging mode (Vigan et al. 2010) with the $\mathrm{H}_{2} \mathrm{H}_{3}$ filters $\left(\lambda_{\mathrm{H}_{2}}=1.593 \pm\right.$ $\left.0.055 \mu \mathrm{m} ; \lambda_{\mathrm{H}_{3}}=1.667 \pm 0.056 \mu \mathrm{m}\right)$, and the IFS integral field spectrograph (Claudi et al. 2008) simultaneously in $Y-J$ $\left(0.95-1.35 \mu \mathrm{m}, R_{\lambda}=54\right)$ mode. The registration of the star position behind the coronagraph and the point spread function were taken at the beginning and the end of the sequence. In the deep coronagraphic images, four faint candidates were detected within 7 " of HIP 65426. The companion candidate located at about 830 mas and position angle of $150^{\circ}$ (hereafter cc-0) revealed promising photometric properties and had a peculiar position in the color-magnitude diagrams used to rank the SHINE candidates. The source was then re-observed on February 7 th,

HARPS Program ID 098.C-0739(A).
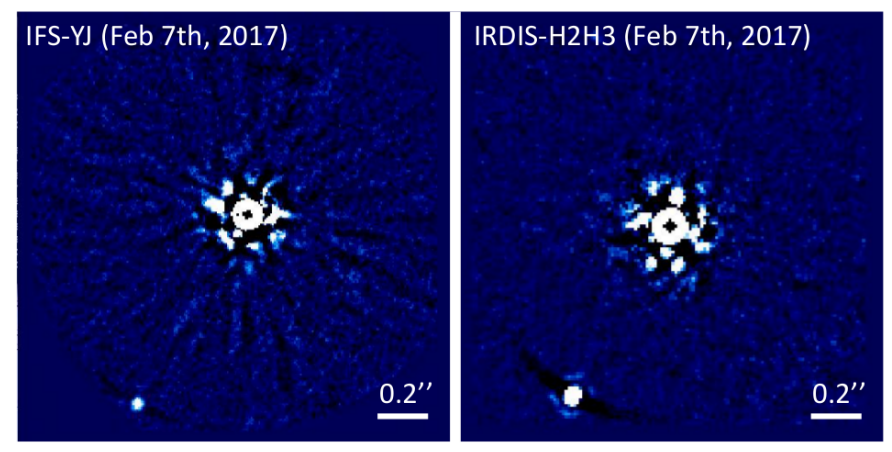

Fig. 1. Left: IFS $Y J$-band TLOCI image of HIP 65426 A and b from February 7th 2017. The planet is well detected at a separation of $830 \pm$ 3 mas and position angle of $150.0 \pm 0.3 \mathrm{deg}$ from HIP 65426. Right: IRDIS $\mathrm{H}_{2} \mathrm{H}_{3}$ combined TLOCI image of HIP $65426 \mathrm{~A}$ and $\mathrm{b}$ for the same night. In both images, north is up and east is left.

2017, with the same IRDIFS mode to test that this close candidate is comoving with HIP 65426. On February 9th, 2017, the IRDIFS-EXT mode was used with IRDIS in the $K_{1} K_{2}$ filters $\left(\lambda_{K_{1}}=2.1025 \pm 0.1020 \mu \mathrm{m} ; \lambda_{K_{2}}=2.2550 \pm 0.1090 \mu \mathrm{m}\right)$ and IFS in $Y-H\left(0.97-1.66 \mu \mathrm{m}, R_{\lambda}=30\right)$ to further constrain its physical and spectral properties. The details of the observing settings and conditions at all epochs are described in Table E.1. To calibrate the IRDIS and IFS datasets, an astrometric field 47 Tuc was observed. The platescale and True North correction solution at each epoch are reported in Table E.1. They are derived from the long-term analysis of the SHINE astrometric calibration described by Maire et al. (2016).

All IRDIS and IFS datasets were reduced at the SPHERE Data Center ${ }^{2}$ (DC) using the SPHERE Data Reduction and Handling (DRH) automated pipeline (Pavlov et al. 2008). Basic corrections for bad pixels, dark current, flat field were applied. For IFS, the SPHERE-DC complemented the DRH pipeline with additional steps that improve the wavelength calibration and the cross-talk and bad pixel correction (Mesa et al. 2015). The products were then used as input to the SHINE Specal pipeline which applies anamorphism correction and flux normalization, followed by different angular and spectral differential imaging algorithms (Galicher et al., in prep.). For the February 7th, 2017 and February 9th, 2017 datasets, we took advantage of the waffle-spot registration to apply a frame-to-frame recentering. The TLOCI (Marois et al. 2014) and PCA (Soummer et al. 2012; Amara \& Quanz 2012) algorithms were specifically used on angular differential imaging data (i.e., without applying any combined spectral differential processing) given the relatively high $\mathrm{S} / \mathrm{N}$ (10-30 in the individual IFS channels, $\geq 50$ with IRDIS) detection of cc-0. Its position and spectrophotometry were extracted using injected fake planets and planetary signature templates to take into account any biases related to the data processing. Both algorithms gave consistent results. The resulting extracted TLOCI images from IFS and IRDIS for the February 7th, 2017 epoch are shown in Fig. 1.

\section{Results}

\subsection{Companionship confirmation}

To test the physical association of cc-0, multi-epoch measurements showing a shared motion (if non-negligible) with the stellar host is a first robust diagnostic before resolving orbital

\footnotetext{
2 http://sphere.osug.fr
} 


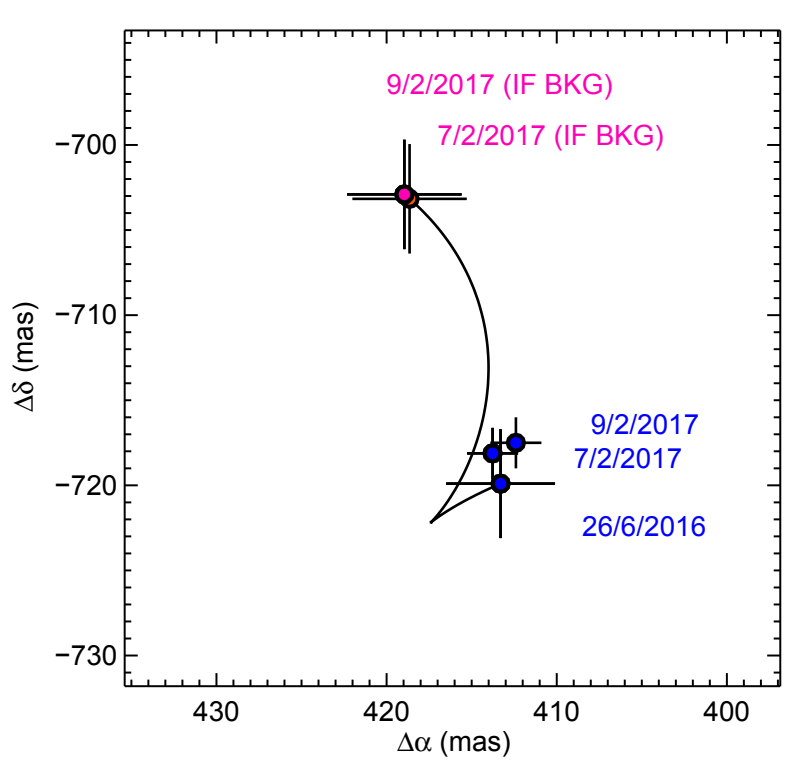

Fig. 2. IRDIS multi-epoch measurements of the position of HIP $65426 \mathrm{~b}$ relative to HIP 65426 in blue from June 26th, 2016 and February 7th, 2017 in $\mathrm{H}_{2}$ and February 9th, 2017 in $K_{1}$. Predictions of the relative position of a stationary background contaminant for the same observing epochs are shown in pink and in black for the continuous evolutive predictions in time.

motion. We therefore used the IRDIS observations of June 26th, 2016, and February 7th and 9th, 2017. We did not consider the first observation of the May 30th 2016 epoch which was acquired under unstable conditions. The astrometric uncertainties are derived at each epoch by quadratically summing errors from the stellar position calibrated with the waffle-spots, the candidate extracted position with Specal and the uncertainties related to the initial pupil-offset rotation, the platescale and True North correction, the anamorphism and the IRDIS dithering. The photometric results obtained with both IRDIS and IFS are given in Table F.1 considering the SPHERE filter transmissions ${ }^{3}$. The astrometric results obtained with IRDIS are reported in Table F.1 and in Fig. 2. Consistent astrometric results were found with IFS. They both unambiguously confirm that cc-0 is not a stationary background contaminant but is comoving with HIP 65426 and is therefore likely a planet (hereafter HIP 65426 b). One additional close candidate (cc-1) located at 2495 mas provides a robust control of our astrometric analysis and is confirmed as a stationary background contaminant. The nature of the two other candidates (cc-2 and cc-3) at larger separations remains to be clarified (considering the astrometric error bars), but their position in the color-magnitude diagram indicates that they are very likely background objects (see Fig. F.1). The relative astrometry and photometry for these additional candidates are reported in Table F.2 for June 26th, 2016. No significant orbital motion for HIP 65426 b is measured in the present data. This is consistent with the expected long orbital period $(P \sim 600 \mathrm{yr}$ for a circular orbit with semi-major axis equal to the projected separation). Finally, the probability of having at least one background contaminant of similar brightness or brighter within the separation of HIP $65426 \mathrm{~b}$ and with proper motion less than $5 \sigma$ deviant from the HIP 65426 proper motion is less than $1 \%$ given the galactic coordinates of HIP 65426 and the predicted space and velocity

\footnotetext{
3 https://www.eso.org/sci/facilities/paranal/ instruments/sphere/inst/filters.html
}

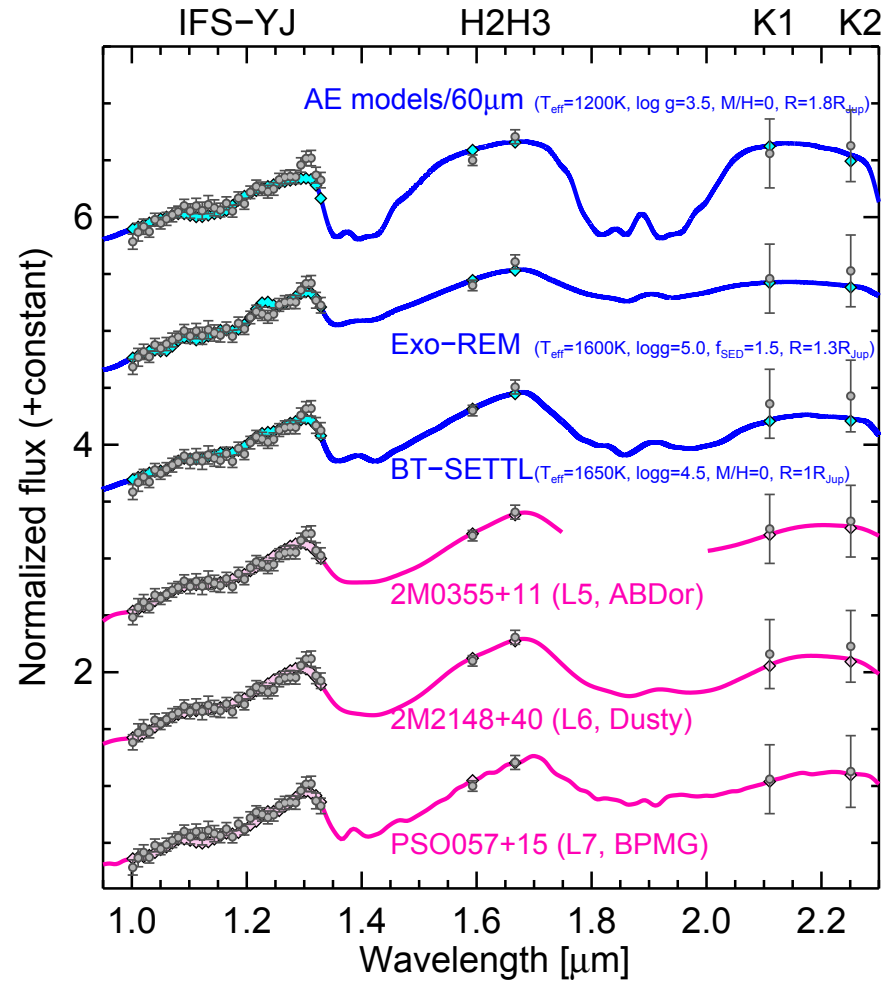

Fig. 3. Near-infrared spectrum of HIP 65426 b extracted with TLOCI compared with (i) the best-fit empirical spectra in pink, and (ii) the bestfit model atmosphere from the Exo-REM, PHOENIX BT-Settl-2014 and thick AE cloud atmospheric models in blue.

distribution of field stars from the Galaxia galactic population model of Sharma et al. (2011).

\subsection{Spectral typing analysis}

The TLOCI extracted spectro-photometric measurements of HIP $65426 \mathrm{~b}$ between 0.95 and $2.26 \mu \mathrm{m}$ (converted to physical fluxes using the VOSA ${ }^{4}$ tool) are reported in Fig. 3 . We compared them to a large variety of reference low-resolution spectra of late-M and $\mathrm{L}$ dwarfs compiled from the literature (Burgasser 2014; Best et al. 2015; Mace et al. 2013; Allers \& Liu 2013) as well as spectra of young imaged exoplanets and brown dwarfs close to the L/T transition (Patience et al. 2010; Zurlo et al. 2016; De Rosa et al. 2014; Artigau et al. 2015; Gauza et al. 2015). We considered the $G$ goodness-of-fit indicator defined in Cushing et al. (2008) which accounts for the filter and spectral channel widths to compare each of the template spectra to the spectrophotometric datapoints of HIP $65426 \mathrm{~b}$. The best empirical fits are obtained for the young L5 and L7 dwarfs 2MASS J035523.37+113343.7 and PSO J057.2893+15.2433 recently identified as candidate members of the young, moving groups AB Doradus (50-150 Myr) and $\beta$ Pictoris (20-30 Myr), respectively, (Faherty et al. 2013; Liu et al. 2013; Best et al. 2015) and the dusty L6 dwarf 2MASS J21481628+4003593 (Looper et al. 2008). Figure 3 shows how well they reproduce the near-infrared slope of the spectrum of HIP $65426 \mathrm{~b}$ between 0.95 and $2.26 \mu \mathrm{m}$ as well as the water absorption at $1.33 \mu \mathrm{m}$. This comparison confirms a low-surface-gravity atmosphere of spectral type L $6 \pm 1$ for HIP 65426 b consistent with a young massive planet at the age of the LCC.

\footnotetext{
4 http://svo2.cab.inta-csic.es/theory/vosa/
} 


\subsection{Physical properties}

Using a bolometric correction derived from the ones found by Filippazzo et al. (2015) for the dusty L5 to L7.5 dwarfs 2MASS J21481628+4003593， 2MASS J035523.37+113343.7, PSO J318.5338-22.8603, and WISEP J004701.06+680352.1, we derive a bolometric luminosity of $-4.06 \pm 0.10 \mathrm{dex}$ for HIP $65426 \mathrm{~b}$. Considering an age of $14 \pm 4 \mathrm{Myr}$ and a distance of $111.4 \pm 3.8 \mathrm{pc}$, it converts into the following predicted masses, effective temperatures and radii by the Lyon's group hot-start models: $M=7_{-1}^{+2} M_{\mathrm{Jup}}, T_{\mathrm{eff}}=1500_{-200}^{+100} \mathrm{~K}$ and $R=1.5 \pm 0.1 R_{\mathrm{Jup}}$ for the COND03 models (Baraffe et al. 2003) and $M=10 \pm$ $2 M_{\text {Jup }}, T_{\text {eff }}=1500_{-200}^{+100} \mathrm{~K}$ and $R=1.5 \pm 0.1 R_{\text {Jup }}$ for the DUSTY models (Chabrier et al. 2000). Consistent results are found with the models of Mordasini (2013) for the hot-start solutions and higher masses for the warm-start solutions $\left(M=12 M_{\text {Jup }}\right.$, $T_{\text {eff }}=1260 \mathrm{~K}$ and $R=1.3 R_{\text {Jup }}$ ). To further explore the physical properties of HIP $65426 \mathrm{~b}$, we compared our data to the synthetic grids of three atmospheric models previously used in the characterization of the planets around HR 8799 (Bonnefoy et al. 2016). They are the Exo-REM models (Baudino et al. 2015), the 2014 version of the PHOENIX BT-Settl atmospheric models described in Allard (2014) and Baraffe et al. (2015) and the thick AE cloud parametric models of Madhusudhan et al. (2011). The best fits for each grid are reported in Fig. 3. The Exo-REM and PHOENIX BT-Settl models favor an effective temperature of $T_{\text {eff }}=1600_{-200}^{+100} \mathrm{~K}$, slightly higher than the one derived by the semi-empirical scale of Filippazzo et al. (2015) for young L6 $\pm 1\left(T_{\text {eff }}=1200-1400 \mathrm{~K}\right)$, but consistent with the evolutionary model predictions. They however favor high-surface-gravity solutions of $\log (g)=4.0-5.0$ with smaller radii $\left(1.0-1.3 R_{\mathrm{Jup}}\right)$. On the contrary, the thick AE cloud parametric models that predict solutions with $T_{\text {eff }}=1200 \pm 100 \mathrm{~K}$ and $\log (g)=3.5$ reproduce the spectral morphology but predict luminosities which are too low, thus leading to radii above the evolutionary model predictions $\left(\sim 1.8 R_{\text {Jup }}\right)$.

The inferred chemical and physical properties of HIP 65426 b place this new planet in a very interesting sequence of young brown dwarfs and exoplanets discovered in the 5-20 Myr-old Scorpius-Centaurus association (hereafter Sco-Cen). It is lighter and cooler than the late-M brown dwarf companions discovered by Aller et al. (2013) and Hinkley et al. (2015) and the massive planetary mass companions GSC 06214$00210 \mathrm{~b}\left(14-17 M_{\text {Jup }}\right.$ at $320 \mathrm{au}$; M9 at $5 \mathrm{Myr}$; Lachapelle et al. 2015; Ireland et al. 2011), UScoCTIO 108 b (6-16 $M_{\text {Jup }}$ at $670 \mathrm{au}$; M9.5 at $5 \mathrm{Myr}$; Bonnefoy et al. 2014; Béjar et al. 2008), HD $106906 \mathrm{~b}\left(11 M_{\text {Jup }}\right.$ at $650 \mathrm{au}$; L2.5 at $13 \mathrm{Myr}$; Bailey et al. 2014) and 1RXS J160929.1-210524 b (7-12 $M_{\text {Jup }}$ at $330 \mathrm{au}$; L2-4 at 5 Myr; Lachapelle et al. 2015; Manjavacas et al. 2014; Lafrenière et al. 2008). On the other hand, HIP 65426 b is probably more massive and hotter than the planet HD 95086 b (4-5 $M_{\text {Jup }}$ at 56 au, L8-type, 17 Myr; Rameau et al. 2013). This spectral and physical sequence is particularly interesting to study the main phase of transitions occurring in the atmosphere of brown dwarfs and exoplanets and influencing their spectra and luminosity, such as the formation of clouds and their properties as a function of particle size, composition, and location in the atmosphere or the role of non-equilibrium chemistry processes. Further characterization in the thermal-infrared domain with JWST or ground-based instrument like $\mathrm{NaCo}$ will allow us to explore in more detail the young planetary atmosphere of HIP 65426 b. Dedicated photometric variability monitoring would also be opportune as HIP 65426 b shares a similar spectral type and young age with the two highest-amplitude (7-10\%)
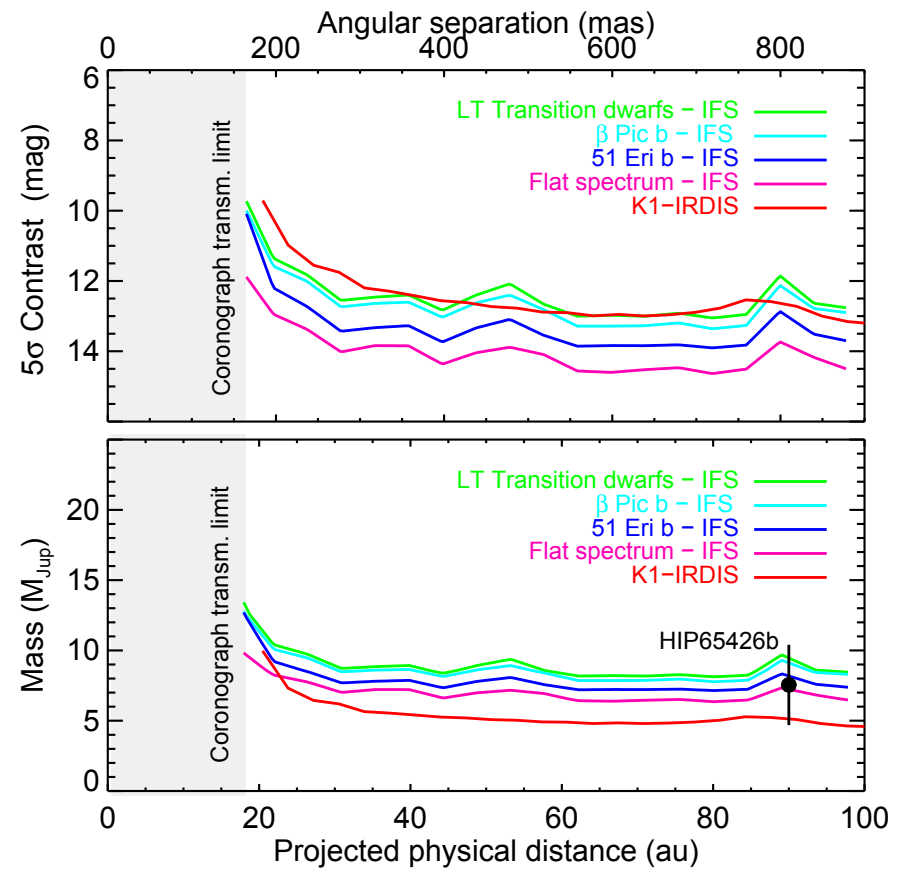

Fig. 4. Top: SPHERE IFS and IRDIS $5 \sigma$ detection limits as a function of the angular separation taken from February 7th and 9th, 2017. Bottom: SPHERE IFS and IRDIS $5 \sigma$ detection limits converted in terms of masses using DUSTY model predictions as a function of the projected physical separation. For IFS, different spectral energy distributions were considered for the injected planets to explore the impact of the flux loss cancellation and different planet properties in the final detection limits. Contrast curves were cut at $0.15^{\prime \prime}$ because of the low transmission of the coronagraph. The location and the predicted mass by the DUSTY models of HIP $65426 \mathrm{~b}$ are reported.

variable L-type dwarfs known (PSO J318.5-22, L7 member of $\beta$ Pic, Biller et al. 2015; Liu et al. 2013; and WISE0047, L6.5 member of AB Dor, Lew et al. 2016; Gizis et al. 2012) and, as radial velocity measurements suggest, we may be observing the system close to edge-on.

\section{Discussion}

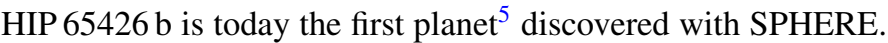
The planet is orbiting at a relatively large projected physical distance of about $92 \mathrm{au}$ from the intermediate-mass primary HIP 65426. Contrary to most of the young, intermediate-mass stars hosting an imaged planet, no evidence of a debris disk, a remnant planetesimal belt, has been found for HIP 65426. The analysis of the optical to mid-infrared photometry shows that, if the star is still hosting a debris disk, it would be located at distances larger than 100 au (i.e. farther out than the planet location) and with an upper limit to the micron-sized dust mass of $3.2 \times 10^{-4} M_{\oplus}$ (see Appendix D). No signs of multiplicity have been observed so far for HIP 65426, which could have explained a rapid dispersal of the primordial protoplanetary disk, but this should still be investigated. Another intriguing aspect of the system is that HIP 65426 is an extremely fast rotator as evidenced by our HARPS observations. No similar cases are known among the Sco-Cen and young, nearby intermediate-mass association

\footnotetext{
5 With a mass ratio to its host of $q=0.004$, we consider HIP $65426 \mathrm{~b}$ as a planet as suggested by the local minimum observed for the mass-ratio distribution of low-mass companions orbiting Sun-like stars (Sahlmann et al. 2011; Reggiani et al. 2016).
} 
members, nor among the intermediate-mass primaries hosting young imaged giant planets (see Appendix B). Although fast stellar rotation is consistent with the picture of a rapid disk dispersal disabling disk-braking, planetary formation must have time to occur to explain the formation of HIP $65426 \mathrm{~b}$. The planet location would not favor a formation by core accretion unless HIP $65426 \mathrm{~b}$ formed significantly closer to the star followed by a planet-planet scattering event. An increase of angular momentum by engulfing an inner massive scatterer could explain the fast rotation of HIP 65426, but this remains to be tested by dedicated simulations. From our observations, we cannot exclude the presence of unseen inner massive planets in that system that could have scattered out HIP 65426 b. However, our current detection limits set relatively good constraints on their possible masses ( $\leq 5 M_{\text {Jup }}$ beyond $20 \mathrm{au}$ ), as shown in Fig. 4. As a consequence of a scattering event, the orbit of HIP $65426 \mathrm{~b}$ would also be rather eccentric which could be probed with further astrometric monitoring. If formed in-situ at its current location, formation by disk instability would be a better alternative, which would be consistent with the metallicity of the host star not enhanced with respect to the solar value. Finally, the formation of an extreme mass-ratio binary by gravo-turbulent fragmentation (Hennebelle \& Chabrier 2011) cannot be excluded.

Acknowledgements. We acknowledge financial support from the Programme National de Planétologie (PNP) and the Programme National de Physique Stellaire (PNPS) of CNRS-INSU. This work has also been supported by a grant from the French Labex OSUG@2020 (Investissements d'avenir - ANR10 LABX56). The project is supported by CNRS, by the Agence Nationale de la Recherche (ANR-14-CE33-0018). This work has made use of the SPHERE Data Centre, jointly operated by OSUG/IPAG (Grenoble), PYTHEAS/LAM/CESAM (Marseille), OCA/Lagrange (Nice) and Observtoire de Paris/LESIA (Paris) We thank P. Delorme and E. Lagadec (SPHERE Data Centre) for their efficient help during the data reduction process. SPHERE is an instrument designed and built by a consortium consisting of IPAG (Grenoble, France), MPIA (Heidelberg, Germany), LAM (Marseille, France), LESIA (Paris, France), Laboratoire Lagrange (Nice, France), INAF-Osservatorio di Padova (Italy), Observatoire de Genève (Switzerland), ETH Zurich (Switzerland), NOVA (The Netherlands), ONERA (France) and ASTRON (The Netherlands) in collaboration with ESO. SPHERE was funded by ESO, with additional contributions from CNRS (France), MPIA (Germany), INAF (Italy), FINES (Switzerland) and NOVA (The Netherlands). SPHERE also received funding from the European Commission Sixth and Seventh Framework Programmes as part of the Optical Infrared Coordination Network for Astronomy (OPTICON) under grant number RII3-Ct-2004-001566 for FP6 (2004-2008), grant number 226604 for FP7 (2009-2012) and grant number 312430 for FP7 (2013-2016). M.B. thanks A. Best, K. Allers, G. Mace, E. Artigau, B. Gauza, R. D. Rosa M.-E. Naud, F.-R. Lachapelle, J. Patience, J. Gizis, A. Burgasser, M. Liu, A. Schneider, K. Aller, B. Bowler, S. Hinkley, and K. Kellogg for providing their spectra of young, brown dwarf companions. This publication makes use of VOSA, developed under the Spanish Virtual Observatory project supported from the Spanish MICINN through grant AyA2011-24052. This research has benefitted from the SpeX Prism Spectral Libraries, maintained by A. Burgasser at http://pono.ucsd.edu/ adam/browndwarfs/spexprism. This research has made use of the Washington Double Star Catalog maintained at the US Naval Observatory. This work has made use of data from the European Space Agency (ESA) mission Gaia (https://www.cosmos.esa.int/gaia), processed by the Gaia Data Processing and Analysis Consortium (DPAC, https //www.cosmos.esa.int/web/gaia/dpac/consortium). Part of this work has been carried out within the frame of the National Centre for Competence in Research PlanetS supported by the Swiss National Science Foundation (SNSF). MRM, HMS, and SD are pleased to acknowledge this financial support of the SNSF.

\section{References}

Allard, F. 2014, in Exploring the Formation and Evolution of Planetary Systems, eds. M. Booth, B. C. Matthews, \& J. R. Graham, IAU Symp., 299, 271

Aller, K. M., Kraus, A. L., Liu, M. C., et al. 2013, ApJ, 773, 63

Allers, K. N., \& Liu, M. C. 2013, ApJ, 772, 79

Amara, A., \& Quanz, S. P. 2012, MNRAS, 427, 948

Artigau, É., Gagné, J., Faherty, J., et al. 2015, ApJ, 806, 254

Bailey, V., Meshkat, T., Reiter, M., et al. 2014, ApJ, 780, L4
Baraffe, I., Chabrier, G., Barman, T. S., Allard, F., \& Hauschildt, P. H. 2003, A\&A, 402, 701

Baraffe, I., Homeier, D., Allard, F., \& Chabrier, G. 2015, A\&A, 577, A42

Baudino, J.-L., Bézard, B., Boccaletti, A., et al. 2015, A\&A, 582, A83

Bayo, A., Rodrigo, C., Barrado Y Navascués, D., et al. 2008, A\&A, 492, 277

Béjar, V. J. S., Zapatero Osorio, M. R., Pérez-Garrido, A., et al. 2008, ApJ, 673, L185

Best, W. M. J., Liu, M. C., Magnier, E. A., et al. 2015, ApJ, 814, 118

Beuzit, J.-L., Feldt, M., Dohlen, K., et al. 2008, in Ground-based and Airborne Instrumentation for Astronomy II, Proc. SPIE, 7014, 701418

Biller, B. A., Vos, J., Bonavita, M., et al. 2015, ApJ, 813, L23

Bonnefoy, M., Chauvin, G., Lagrange, A.-M., et al. 2014, A\&A, 562, A127

Bonnefoy, M., Zurlo, A., Baudino, J. L., et al. 2016, A\&A, 587, A58

Bowler, B. P. 2016, PASP, 128, 102001

Bressan, A., Marigo, P., Girardi, L., et al. 2012, MNRAS, 427, 127

Burgasser, A. J. 2014, in Astronomical Society of India Conference Series, 11

Burns, J. A., Lamy, P. L., \& Soter, S. 1979, Icarus, 40, 1

Carbillet, M., Bendjoya, P., Abe, L., et al. 2011, Exp. Astron., 30, 39

Chabrier, G., Baraffe, I., Allard, F., \& Hauschildt, P. 2000, ApJ, 542, 464

Chen, C. H., Pecaut, M., Mamajek, E. E., Su, K. Y. L., \& Bitner, M. 2012, ApJ, 756,133

Claudi, R. U., Turatto, M., Gratton, R. G., et al. 2008, in Ground-based and Airborne Instrumentation for Astronomy II, Proc. SPIE, 7014, 70143E

Cutri, R. M., Skrutskie, M. F., van Dyk, S., et al. 2003, 2MASS All Sky Catalog of point sources, http://irsa.ipac.caltech.edu/ applications/Gator/

David, T. J., \& Hillenbrand, L. A. 2015, ApJ, 804, 146

De Rosa, R. J., Patience, J., Ward-Duong, K., et al. 2014, MNRAS, 445, 3694

de Zeeuw, P. T., Hoogerwerf, R., de Bruijne, J. H. J., Brown, A. G. A., \& Blaauw, A. 1999, AJ, 117, 354

Dohlen, K., Langlois, M., Saisse, M., et al. 2008, in Ground-based and Airborne Instrumentation for Astronomy II, Proc. SPIE, 7014,70143L

Draine, B. T. 2003, ApJ, 598, 1026

Faherty, J. K., Rice, E. L., Cruz, K. L., Mamajek, E. E., \& Núñez, A. 2013, AJ, 145,2

Fang, Q., Herczeg, G. J., \& Rizzuto, A. 2017, ApJ, 842, 123

Filippazzo, J. C., Rice, E. L., Faherty, J., et al. 2015, ApJ, 810, 158

Gaia Collaboration (Brown, A. G. A., et al.) 2016, A\&A, 595, A2

Gauza, B., Béjar, V. J. S., Pérez-Garrido, A., et al. 2015, ApJ, 804, 96

Gizis, J. E., Faherty, J. K., Liu, M. C., et al. 2012, AJ, 144, 94

Guerri, G., Daban, J.-B., Robbe-Dubois, S., et al. 2011, Exp. Astron., 30, 59

Hennebelle, P., \& Chabrier, G. 2011, ApJ, 743, L29

Hinkley, S., Bowler, B. P., Vigan, A., et al. 2015, ApJ, 805, L10

Ireland, M. J., Kraus, A., Martinache, F., Law, N., \& Hillenbrand, L. A. 2011, ApJ, 726, 113

Jovanovic, N., Guyon, O., Lozi, J., et al. 2016, in Adaptive Optics Systems V, Proc. SPIE, 9909, 99090W

Lachapelle, F.-R., Lafrenière, D., Gagné, J., et al. 2015, ApJ, 802, 61

Lafrenière, D., Jayawardhana, R., \& van Kerkwijk, M. H. 2008, ApJ, 689, L153

Lew, B. W. P., Apai, D., Zhou, Y., et al. 2016, ApJ, 829, L32

Liu, M. C., Magnier, E. A., Deacon, N. R., et al. 2013, ApJ, 777, L20

Looper, D. L., Kirkpatrick, J. D., Cutri, R. M., et al. 2008, ApJ, 686, 528

Mace, G. N., Kirkpatrick, J. D., Cushing, M. C., et al. 2013, ApJS, 205, 6

Macintosh, B., Graham, J. R., Ingraham, P., et al. 2014, Proc. Nat. Acad. Sci., 111,12661

Macintosh, B., Graham, J. R., Barman, T., et al. 2015, Science, 350, 64

Maire, A.-L., Langlois, M., Dohlen, K., et al. 2016, in Proc. SPIE Conf. Ser., 9908, 990834

Manjavacas, E., Bonnefoy, M., Schlieder, J. E., et al. 2014, A\&A, 564, A55

Marois, C., Correia, C., Véran, J.-P., \& Currie, T. 2014, in Exploring the Formation and Evolution of Planetary Systems, eds. M. Booth, B. C. Matthews, \& J. R. Graham, IAU Symp, 299, 48

Mason, B. D., Wycoff, G. L., Hartkopf, W. I., Douglass, G. G., \& Worley, C. E. 2001, AJ, 122, 3466

Mayor, M., Pepe, F., Queloz, D., et al. 2003, The Messenger, 114, 20

Mordasini, C. 2013, A\&A, 558, A113

Olofsson, J., Samland, M., Avenhaus, H., et al. 2016, A\&A, 591, A108

Patience, J., King, R. R., de Rosa, R. J., \& Marois, C. 2010, A\&A, 517, A76

Pavlov, A., Möller-Nilsson, O., Feldt, M., et al. 2008, in Advanced Software and Control for Astronomy II, Proc. SPIE, 7019, 701939

Pecaut, M. J., \& Mamajek, E. E. 2013, ApJS, 208, 9

Pecaut, M. J., \& Mamajek, E. E. 2016, MNRAS, 461, 794

Perryman, M. A. C., Lindegren, L., Kovalevsky, J., et al. 1997, A\&A, 323, L49

Rameau, J., Chauvin, G., Lagrange, A.-M., et al. 2013, ApJ, 772, L15

Reggiani, M., Meyer, M. R., Chauvin, G., et al. 2016, A\&A, 586, A147

Rizzuto, A. C., Ireland, M. J., \& Robertson, J. G. 2011, MNRAS, 416, 3108 
Sahlmann, J., Ségransan, D., Queloz, D., \& Udry, S. 2011, in The Astrophysics of Planetary Systems: Formation, Structure, and Dynamical Evolution, eds. A. Sozzetti, M. G. Lattanzi, \& A. P. Boss, IAU Symp., 276, 117 Sharma, S., Bland-Hawthorn, J., Johnston, K. V., \& Binney, J. 2011, ApJ, 730, 3 Soummer, R., Pueyo, L., \& Larkin, J. 2012, ApJ, 755, L28

Viana Almeida, P., Santos, N. C., Melo, C., et al. 2009, A\&A, 501, 965

Vigan, A., Moutou, C., Langlois, M., et al. 2010, MNRAS, 407, 71

Zorec, J., \& Royer, F. 2012, A\&A, 537, A120

Zurlo, A., Vigan, A., Galicher, R., et al. 2016, A\&A, 587, A57

${ }^{1}$ Univ. Grenoble Alpes, CNRS, IPAG, 38000 Grenoble, France e-mail: gael.chauvin@univ-grenoble-alpes.fr

2 Unidad Mixta Internacional Franco-Chilena de Astronomía, CNRS/INSU UMI 3386 and Departamento de Astronomía, Universidad de Chile, Casilla 36-D, Santiago, Chile

${ }^{3}$ INAF-Osservatorio Astronomico di Padova, Vicolo dell' Osservatorio 5, 35122, Padova, Italy

4 Aix Marseille Université, CNRS, LAM (Laboratoire d'Astrophysique de Marseille) UMR 7326, 13388 Marseille, France

5 CRAL, UMR 5574, CNRS, Université de Lyon, École Normale Supérieure de Lyon, 46 Allée d'Italie, 69364 Lyon Cedex 07, France

6 Max Planck Institute for Astronomy, Königstuhl 17, 69117 Heidelberg, Germany

${ }^{7}$ Institute for Astronomy, ETH Zurich, Wolfgang-Pauli-Strasse 27, 8093 Zurich, Switzerland

8 The University of Michigan, Ann Arbor, MI 48109, USA

${ }_{9}$ Geneva Observatory, University of Geneva, Chemin des Mailettes 51, 1290 Versoix, Switzerland

10 SUPA, Institute for Astronomy, The University of Edinburgh, Royal Observatory, Blackford Hill, Edinburgh, EH9 3HJ, UK

11 LESIA, Observatoire de Paris, PSL Research University, CNRS, Sorbonne Universités, UPMC Univ. Paris 06, Univ. Paris Diderot, Sorbonne Paris Cité, 5 place Jules Janssen, 92195 Meudon, France

12 Instituto de Física y Astronomía, Facultad de Ciencias, Universidad de Valparaíso, Av. Gran Bretaña 1111, Valparaíso, Chile
13 Université Côte d'Azur, OCA, CNRS, Lagrange, France

14 Department of Astronomy, Stockholm University, AlbaNova University Center, 10691 Stockholm, Sweden

15 Physikalisches Institut, University of Bern, Sidlerstrasse 5, 3012 Bern, Switzerland

16 INAF - Catania Astrophysical Observatory, via S. Sofia, 7895123 Catania, Italy

17 Núcleo de Astronomía, Facultad de Ingeniería, Universidad Diego Portales, Av. Ejercito 441, Santiago, Chile

18 Anton Pannekoek Institute for Astronomy, Science Park 904, 1098 XH Amsterdam, The Netherlands

19 INAF-Osservatorio Astrofisico di Arcetri, Largo E. Fermi 5, 50125 Firenze, Italy

20 INAF-Osservatorio Astronomico di Capodimonte, Salita Moiariello 16, 80131 Napoli, Italy

${ }^{21}$ ONERA (Office National d'Études et de Recherches Aérospatiales), BP 72, 92322 Châtillon, France

${ }^{22}$ European Southern Observatory (ESO), Karl-Schwarzschild-Str. 2, 85748 Garching, Germany

${ }^{23}$ NOVA Optical Infrared Instrumentation Group, Oude Hoogeveensedijk 4, 7991 PD Dwingeloo, The Netherlands

24 INAF-Osservatorio Astronomico di Roma, via di Frascati 33, 00040 Monte Porzio Catone, Italy

25 Department of Astrophysics, Denys Wilkinson Building, Keble Road, Oxford, OX1 3RH, UK

${ }^{26}$ European Southern Observatory (ESO), Alonso de Córdova 3107, Vitacura, Casilla 19001, Santiago, Chile

27 Institute of Astronomy, University of Cambridge, Madingley Road, Cambridge CB3 0HA, UK

28 School of Earth \& Space Exploration, Arizona State University, Tempe AZ 85287, USA

29 Ural Federal University, Yekaterinburg 620002, Russia

30 CNRS, CFHT, 65-1238 Mamalahoa Hwy, Kamuela HI 96743, USA

31 Exoplanets and Stellar Astrophysics Laboratory, NASA Goddard Space Flight Center, 8800 Greenbelt Rd., Greenbelt, MD 20771, USA 
Table A.1. Stellar parameters of HIP 65426.

\begin{tabular}{|c|c|c|}
\hline Parameter & Value & References \\
\hline$V(\mathrm{mag})$ & 7.01 & HIPPARCOS $^{a}$ \\
\hline$B-V(\mathrm{mag})$ & 0.093 & HIPPARCOS $^{a}$ \\
\hline$V-I(\mathrm{mag})$ & 0.11 & $\operatorname{HIPPARCOS}^{a}$ \\
\hline$J$ (mag) & $6.826 \pm 0.019$ & $2 \mathrm{MASS}^{b}$ \\
\hline$H$ (mag) & $6.853 \pm 0.049$ & $2 \mathrm{MASS}^{b}$ \\
\hline$K(\mathrm{mag})$ & $6.771 \pm 0.018$ & $2 \mathrm{MASS}^{b}$ \\
\hline Parallax (mas) & $8.98 \pm 0.30$ & Gaia $\mathrm{DR} 1^{c}$ \\
\hline$\mu_{\alpha}\left(\operatorname{mas~yr}^{-1}\right)$ & $-33.923 \pm 0.030$ & Gaia $\mathrm{DR} 1^{c}$ \\
\hline$\mu_{\delta}\left(\operatorname{mas~yr}^{-1}\right)$ & $-18.955 \pm 0.031$ & Gaia $\mathrm{DR} 1^{c}$ \\
\hline $\mathrm{RV}\left(\mathrm{km} \mathrm{s}^{-1}\right)$ & $-5.2 \pm 1.3$ & this paper \\
\hline $\mathrm{SpT}$ & A2V & \\
\hline$T_{\text {eff }}(\mathrm{K})$ & $8840 \pm 200$ & SpT+Pecaut calib. \\
\hline$E(B-V)$ & $0.01 \pm 0.01$ & this paper \\
\hline$v \sin i\left(\mathrm{~km} \mathrm{~s}^{-1}\right)$ & $299 \pm 9$ & this paper \\
\hline Age (Myr) & $14_{-4}^{+4}$ & this paper \\
\hline$M_{\text {star }}\left(M_{\odot}\right)$ & $1.96 \pm 0.04$ & this paper \\
\hline$R_{\text {star }}\left(R_{\odot}\right)$ & $1.77 \pm 0.05$ & this paper \\
\hline
\end{tabular}

References. (a) Perryman et al. (1997), (b) Cutri et al. (2003), (c) Gaia Collaboration (2016).

\section{Appendix A: Isochronal ages of HIP 65426 and neighboring stars}

Adopting the values from Table A.1, the placement in absolute magnitude in $V$-band versus effective temperature diagram and comparison with theoretical models by Bressan et al. (2012) yields an age of 9-10 Myr when adopting the Gaia DR1 parallax (Fig. A.1). This is slightly younger than the commonly adopted age for LCC (17 Myr). However, recent results highlight the existence of significant age differences at various locations within the Sco-Cen sub-groups (Fang et al. 2017; Pecaut \& Mamajek 2016).

To further refine the age estimate, we considered additional stars physically close to HIP 65426 and with similar kinematic parameters. We selected from Gaia DR1 stars within $5^{\circ}$ of HIP 65426, with parallax within 1 mas, and proper motion within

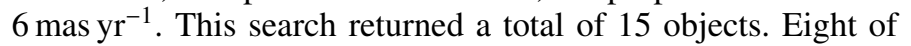
these were previously known as LCC members. The seven stars without RV determination from the literature were observed with FEROS spectrograph at $2.2 \mathrm{~m}$ telescope at La Silla as part of MPIA observing time ${ }^{6}$. Full results of these observations will be presented in a future publication. All the selected stars are probable members of the Sco-Cen group, as resulting from RV, signatures of fast rotation and activity or lithium from data available in the literature or from our FEROS spectra. The maximum space velocity difference with respect to HIP 65426 amounts to $12.9 \mathrm{~km} \mathrm{~s}^{-1}$. Two stars, namely HIP 64044 and TYC 8653-10601, have kinematic values very close to those of HIP 65426 (space velocity difference of 2.1 and $0.9 \mathrm{~km} \mathrm{~s}^{-1}$, respectively). However, being at a projected separation larger than $2.9^{\circ}$ they do not form a bound system with HIP 65426. Figure A.1 shows the absolute magnitude in $V$-band versus effective temperature diagram for HIP 65426 and stars within $5^{\circ}$ with similar distance and kinematics and comparison with theoretical models. Effective temperatures and reddening have been derived from spectral types using the calibration of Pecaut \& Mamajek (2013).

\footnotetext{
6 Program ID 098.A-9007(A).
}

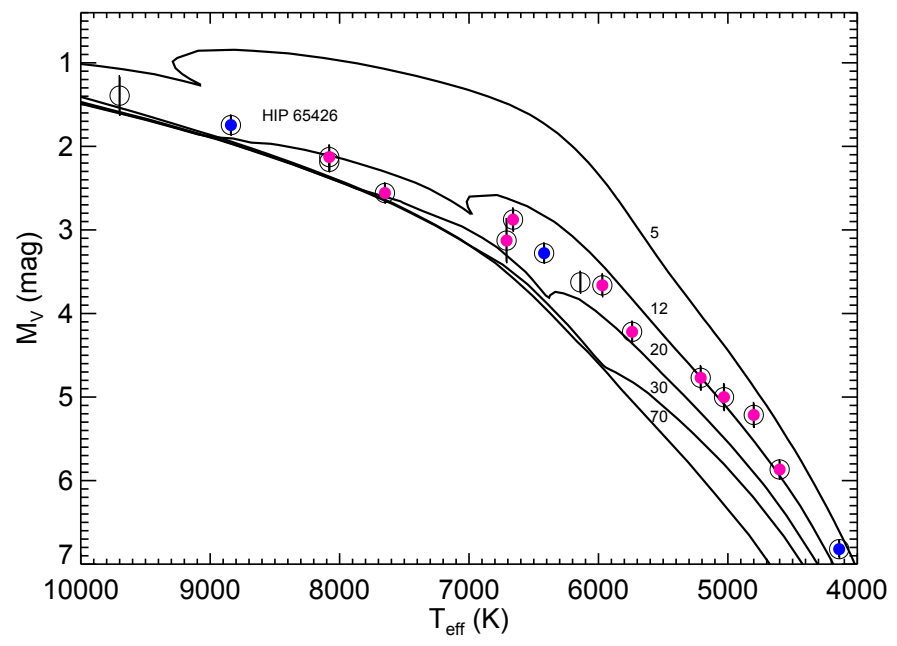

Fig. A.1. Absolute magnitude in $V$-band versus effective temperature diagram for HIP 65426 and stars within $5^{\circ}$ with similar distance and kinematics. Blue circles: HIP 65426 and two stars with space velocity difference smaller than $3 \mathrm{~km} \mathrm{~s}^{-1}$. Red circles: stars with space velocity difference with respect to HIP 65426 between 3 and $10 \mathrm{~km} \mathrm{~s}^{-1}$. Empty circles: stars with space velocity difference larger than $10 \mathrm{~km} \mathrm{~s}^{-1}$. The 5 , 12, 20, 30, 70 Myr solar-metallicity isochrones by Bressan et al. (2012) are overplotted and labeled individually.

The result of this comparison shows that the typical age of LCC stars in the surroundings of HIP 65426 is of the order of 12-16 Myr. This is fully consistent with the Sco-Cen age map by Pecaut \& Mamajek (2016), that yields an age of $14 \mathrm{Myr}$ at the position of HIP 65426 using a complementary approach (much larger number of stars extending to fainter magnitude but relying on kinematic parallaxes, while we used the Gaia trigonometric values). The nominal color-magnitude diagram age of HIP 65426 is younger (9-10 Myr). This is most likely due to the alteration induced by its very fast rotational velocity (David \& Hillenbrand 2015). Therefore, an age of $14 \pm 4 \mathrm{Myr}$ is adopted for this star.

\section{Appendix B: Stellar rotation}

We derived radial and rotational velocity of HIP 65426 from HARPS spectra acquired during the nights of January 16, 17, and 18,2017 . The spectra have a resolution of 115000 and cover the spectral range from 380 to $690 \mathrm{~nm}$. We obtained sequences of two, seven, and five exposures of $5 \mathrm{~min}$ each during the three nights. The spectra were reduced using the HARPS pipeline that provides unidimensional spectra sampled in uniform wavelength steps. We summed the spectra for each night to provide higher $\mathrm{S} / \mathrm{N}$ dataset minimizing the short-term variations due to pulsations.

To test the methodology, we also applied the same procedure to a number of additional A2V stars with archived HARPS spectra. The results make use of a standard cross-correlation function method that exploits a binary mask. We considered two sets of lines: (i) six strong lines ( $\mathrm{Ca}$ II $\mathrm{K}$ and five $\mathrm{H}$ lines from $\mathrm{H} \beta$ to $\mathrm{H} 9$, excluding $\mathrm{H} \epsilon$ ); we used the rotationally broadened core of the lines and (ii) 35 atomic lines. The average radial velocity found $\left(5.2 \pm 1.3 \mathrm{~km} \mathrm{~s}^{-1}\right)$ is close to the literature value $\left(3.1 \pm 1.2 \mathrm{~km} \mathrm{~s}^{-1}\right)$. There is a small difference between the radial velocities in the different dates $\left(\mathrm{rms} \sim 1.3 \mathrm{~km} \mathrm{~s}^{-1}\right)$. However, we think that this result neither supports nor contradicts the hypothesis that HIP 65426 is a binary. We derived a very high projected rotational velocity of $v \sin i=299 \pm 9 \mathrm{~km} \mathrm{~s}^{-1}$. This value is at the 


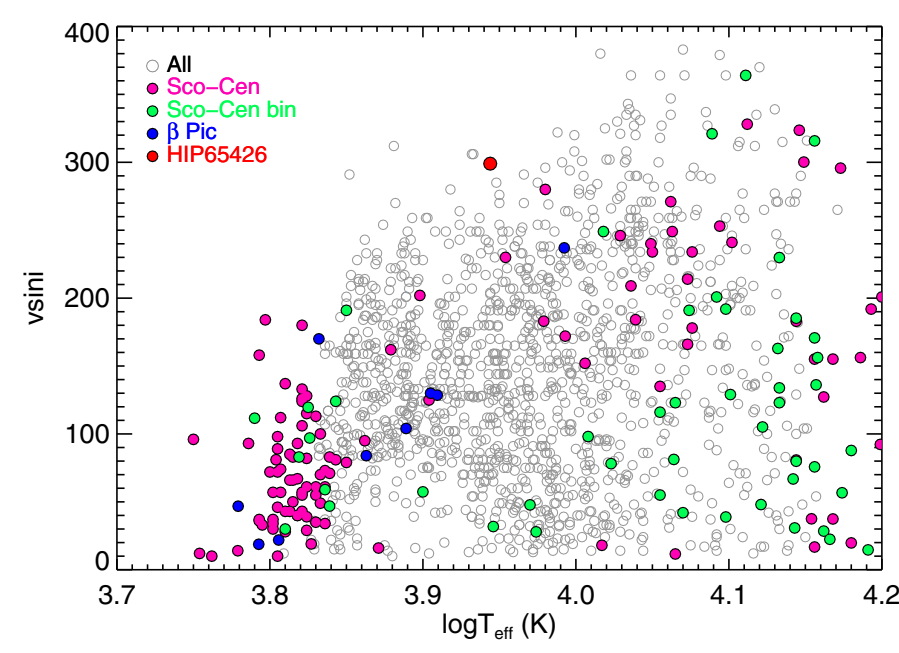

Fig. B.1. Run of the $v \sin i$ values as a function of the effective temperature for "normal" stars in the catalog by Zorec et al. (2012: open gray circles). The red filled circle is HIP 65426. Superimposed are single (pink circles) and binary (green circles) stars in the Sco-Cen association. Stars in the $\beta$ Pic group are also shown (blue filled circles).

upper limit of the distribution of rotational velocities for $\mathrm{A} 2 \mathrm{~V}$ stars. The extracted values of RV and $v \sin i$ are consistent with literature values within the error bars.

If we examine the catalog by Zorec \& Royer (2012), there is no A2V star with $v \sin i>280 \mathrm{~km} \mathrm{~s}^{-1}$ among 119 entries. If we extend the sample by one spectral subclass (A1V-A3V), there is only one A1V star with a $v \sin i>280 \mathrm{~km} \mathrm{~s}^{-1}$ (a close binary) over 368 entries. HIP 65426 is therefore an exceptionally fast rotator. Figure B. 1 compares the $v \sin i$ value for HIP 65426 with values obtained for "normal" stars (i.e., bonafide single stars) in the Zorec et al. (2012) catalog. For comparison, we also plotted single and binary stars in the Sco-Cen association and stars in the $\beta$ Pic group that have similar ages. In these last cases, we also considered $v \sin i$ values from Glebocki et al. (2005) and Chen (2011) catalogs, after correcting them on the same scale as that of the Zorec et al. catalog. The effective temperatures for these stars were obtained from the $B-V$ colours, after calibrating with those from the Zorec et al. (2012) catalog. The peculiar nature of HIP 65426 is quite obvious from this figure. Also, note that the rotational velocities for single late-B and early-A stars in the Sco-Cen association and in the $\beta$ Pic group match well typical values for the "normal" stars in the Zorec et al. (2012) catalog (while binaries typically rotate slower, as also found for field stars). This suggests that stars with masses similar or greater than HIP 65426 have already reached the zero age main sequence in these associations. HIP 65426 is exceptional with respect to both the Sco-Cen association and the field star population.

\section{Appendix C: Binarity}

HIP 65426 is listed as a close visual binary (separation 0.15 $0.3 \operatorname{arcsec}, \Delta \sim 0.1 \mathrm{mag}$ ) in the Washington Double Star Catalog (Mason et al. 2001). We retrieved the individual measurements (kindly provided by Dr. B. Mason), that consist in seven entries between 1926 to 1933 followed by a series of nondetections. There are no indications of close companions in the SPHERE images, including the non-coronagraphic sequence used for photometric calibrations, allowing us to rule out the presence of equal-luminosity companions down to a projected separation of 40 mas. Furthermore, the HARPS spectra do not

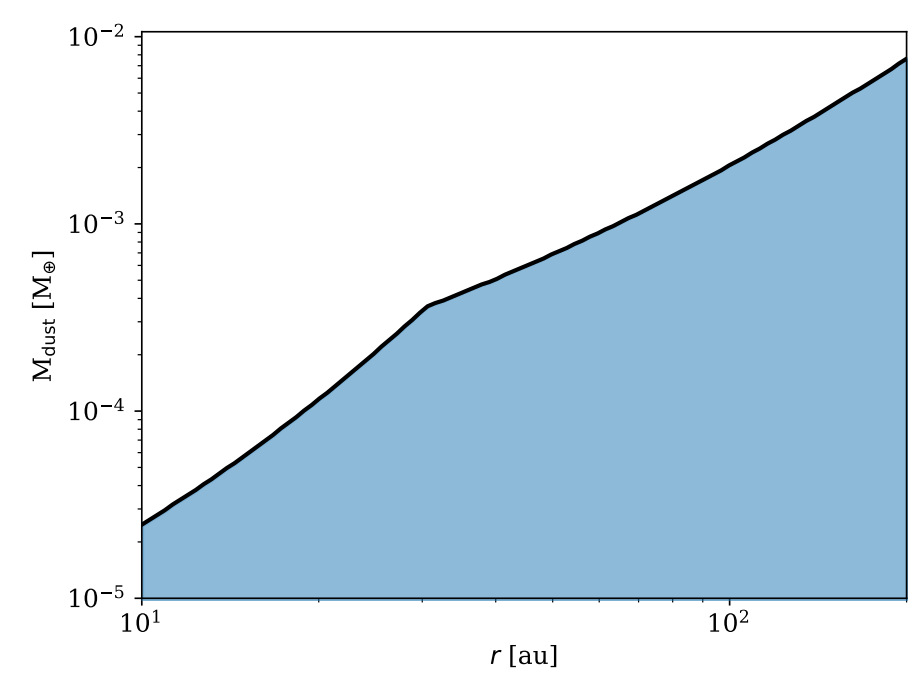

Fig. D.1. The blue-shaded area shows the region in the $r$ - $M_{\text {dust }}$ plane, in which a debris disk could be present and remain compatible with the mid- and far-IR observations.

show indications of multiple components. Finally, an equalluminosity binary would imply an unphysical position on colormagnitude diagram below main sequence. We thus consider it plausible that the previously claimed detection is spurious and consider that we do not find any sign of binarity for HIP 65426.

\section{Appendix D: Upper limits on the dust mass}

Chen et al. (2012) reported a non-detection of any mid-IR excess around HIP 65426. They reported a Spitzer/MIPS upper limit at $70 \mu \mathrm{m}$ of $11.4 \mathrm{mJy}$. Given that the star is young, and that this upper limit does not reach the photospheric flux $(\sim 1.5 \mathrm{mJy})$, we try to estimate the upper limit for the dust mass around HIP 65426. We gather the optical to mid-IR photometry of the star using $\operatorname{VOSA}^{7}$ (Bayo et al. 2008). For the given stellar luminosity and mass $\left(17.3 L_{\odot}\right.$ and $1.95 M_{\odot}$, respectively) we estimate the size of dust grains that would still be on bound orbits around the star. We use optical constant of astro-silicates (Draine 2003), and we compute the radiation pressure to gravitational forces $\beta$ ratio as in Burns et al. (1979). We find that for this composition, grains larger than $s_{\text {blow }} \sim 3.1 \mu \mathrm{m}$ should remain on bound orbits around the star. To estimate the possible configurations for a debris disk to remain compatible with the mid- and far-IR observations, we compute a series of disk models (similar to, Olofsson et al. 2016). We consider a grain size distribution of the form $\mathrm{d} n(s) \propto s^{-3.5} \mathrm{~d} s$, between $s_{\text {min }}=s_{\text {blow }}$ and $s_{\max }=1 \mathrm{~mm}$. We sample $100 r_{\mathrm{i}}$ values for the radial distance between 10 and $200 \mathrm{au}$. For each $r_{\mathrm{i}}$, we consider a disk model between $0.9 \times r_{\mathrm{i}} \leq r_{\mathrm{i}} \leq 1.1 \times r_{\mathrm{i}}$. We then slowly increase the mass of the disk until the thermal emission (plus the stellar contribution) is larger than either the WISE/W4 $22 \mu \mathrm{m}$ point or the Spitzer/MIPS $70 \mu \mathrm{m}$ point. With this exercise we can therefore delimit a region in the $r-M_{\text {dust }}$ plane where debris disks could exist and remain undetected with the current observations (see Fig. D.1). Overall, with our assumptions on the radial extent of the debris disk, we find that it would have to be less massive that $10^{-3} M_{\oplus}$ at about 100 au from the star.

7 http://svo2.cab.inta-csic.es/theory/vosa/ 


\section{Appendix E: Observing Log}

Table E.1. Obs Log of VLT/SPHERE observations.

\begin{tabular}{|c|c|c|c|c|c|c|c|c|c|c|}
\hline UT Date & Instr. & Filter & $\begin{array}{l}\mathrm{DIT}^{a} \\
(\mathrm{~s})\end{array}$ & $N_{\exp }^{a}$ & $\begin{array}{l}\Delta \pi^{a} \\
\left({ }^{\circ}\right)\end{array}$ & $\begin{array}{l}\omega^{a} \\
(")\end{array}$ & $\begin{array}{l}\text { Strehl }^{a} \\
@ 1.6 \mu \mathrm{m}\end{array}$ & Airm. & $\begin{array}{l}\text { True North correction } \\
\left({ }^{\circ}\right)\end{array}$ & $\begin{array}{l}\text { Platescale } \\
\text { (mas/pixel) }\end{array}$ \\
\hline $\begin{array}{l}30-05-2016 \\
30-05-2016\end{array}$ & $\begin{array}{l}\text { IFS } \\
\text { IRDIS }\end{array}$ & $\begin{array}{l}\mathrm{YJ} \\
\mathrm{H} 2 / \mathrm{H} 3\end{array}$ & $\begin{array}{l}64 \\
64\end{array}$ & $\begin{array}{l}60 \\
60\end{array}$ & 34.2 & 0.62 & 0.60 & 1.12 & $\begin{array}{l}-102.20 \pm 0.12 \\
-1.72 \pm 0.06\end{array}$ & $\begin{array}{l}7.46 \pm 0.02 \\
12.255 / 12.251^{b} \pm 0.009\end{array}$ \\
\hline $\begin{array}{l}26-06-2016 \\
26-06-2016\end{array}$ & $\begin{array}{l}\text { IFS } \\
\text { IRDIS }\end{array}$ & $\begin{array}{l}\mathrm{YJ} \\
\mathrm{H} 2 / \mathrm{H} 3\end{array}$ & $\begin{array}{l}64 \\
64\end{array}$ & $\begin{array}{l}75 \\
75\end{array}$ & 42.2 & 0.53 & 0.66 & 1.13 & $\begin{array}{l}-102.25 \pm 0.12 \\
-1.77 \pm 0.05\end{array}$ & $\begin{array}{l}7.46 \pm 0.02 \\
12.255 / 12.251^{b} \pm 0.009\end{array}$ \\
\hline $\begin{array}{l}07-02-2017 \\
07-02-2017\end{array}$ & $\begin{array}{l}\text { IFS } \\
\text { IRDIS }\end{array}$ & $\begin{array}{l}\mathrm{YJ} \\
\mathrm{H} 2 / \mathrm{H} 3\end{array}$ & $\begin{array}{l}64 \\
64\end{array}$ & $\begin{array}{l}80 \\
80\end{array}$ & 44.2 & 0.38 & 0.84 & 1.13 & $\begin{array}{l}-102.19 \pm 0.12 \\
-1.71 \pm 0.05\end{array}$ & $\begin{array}{l}7.46 \pm 0.02 \\
12.255 / 12.251^{b} \pm 0.009\end{array}$ \\
\hline $\begin{array}{l}09-02-2017 \\
09-02-2017\end{array}$ & $\begin{array}{l}\text { IFS } \\
\text { IRDIS }\end{array}$ & $\begin{array}{l}\mathrm{YJH} \\
\mathrm{K} 1 / \mathrm{K} 2\end{array}$ & $\begin{array}{l}64 \\
64\end{array}$ & $\begin{array}{l}80 \\
80\end{array}$ & 49.1 & 0.45 & 0.76 & 1.14 & $\begin{array}{l}-102.19 \pm 0.12 \\
-1.71 \pm 0.05\end{array}$ & $\begin{array}{l}7.46 \pm 0.02 \\
12.267 / 12.263^{b} \pm 0.009\end{array}$ \\
\hline
\end{tabular}

Notes. ${ }^{(a)}$ DIT refers to the integration time, $N_{\text {exp }}$ to the total number of frames in the final mastercube, $\Delta \pi$ to the parallactic angle range during the sequence. The seeing $(\omega)$ and the Strehl ratio conditions were calculated by the SPHERE extreme-adaptive optics system. ${ }^{(b)}$ The two values of platescale correpond to the platescale estimated for both dual-band filters indicated in the Filter column.

\section{Appendix F: Astrometric and photometric detailed results}

Table F.1. IRDIS relative astrometric measurements of HIP 65426 b to HIP 65426 and IRDIS and IFS relative photometric contrast and absolute magnitudes for HIP 65426 b.

\begin{tabular}{cccc}
\hline \hline UT Date & Filter & Separation (mas) & PA $(\mathrm{deg})$ \\
\hline $30 / 05 / 2016$ & $\mathrm{H}_{2}$ & $830.4 \pm 4.9$ & $150.28 \pm 0.22$ \\
$26 / 06 / 2016$ & $\mathrm{H}_{2}$ & $830.1 \pm 3.2$ & $150.14 \pm 0.17$ \\
$07 / 02 / 2017$ & $\mathrm{H}_{2}$ & $827.6 \pm 1.5$ & $150.11 \pm 0.15$ \\
$09 / 02 / 2017$ & $K_{1}$ & $828.8 \pm 1.5$ & $150.05 \pm 0.16$ \\
& & $\Delta(\mathrm{mag})$ & $M_{\text {abs }}(\mathrm{mag})$ \\
\cline { 3 - 4 } $07 / 02 / 2017$ & $J_{2 \text { MASS }}$ & $12.67 \pm 0.40$ & $14.26 \pm 0.42$ \\
& $J_{\text {IFS }}$ & $12.18 \pm 0.08$ & $13.78 \pm 0.11$ \\
$07 / 02 / 2017$ & $H_{2}$ & $11.14 \pm 0.05$ & $12.76 \pm 0.11$ \\
$07 / 02 / 2017$ & $H_{3}$ & $10.78 \pm 0.06$ & $12.39 \pm 0.12$ \\
$09 / 02 / 2017$ & $K_{1}$ & $10.01 \pm 0.31$ & $11.55 \pm 0.33$ \\
$09 / 02 / 2017$ & $K_{2}$ & $9.69 \pm 0.31$ & $11.22 \pm 0.33$ \\
\hline
\end{tabular}

Notes. The composite $J_{\mathrm{IFS}}$-band is estimated between 1.20 and $1.32 \mu \mathrm{m}$.

Table F.2. IRDIS $\mathrm{H}_{2}$ relative astrometric and photometric measurements of June 26th, 2016 for the additional companion candidates in the field.

\begin{tabular}{cccc}
\hline \hline Cand. & Separation (mas) & PA $(\mathrm{deg})$ & $\Delta(\mathrm{mag})$ \\
\hline cc-1 & $2494.7 \pm 12.2$ & $117.42 \pm 0.28$ & $15.6 \pm 0.2$ \\
cc-2 & $3973.1 \pm 14.4$ & $307.91 \pm 0.23$ & $16.2 \pm 0.5$ \\
cc-3 & $6752.6 \pm 11.3$ & $304.28 \pm 0.15$ & $14.1 \pm 0.2$ \\
\hline
\end{tabular}

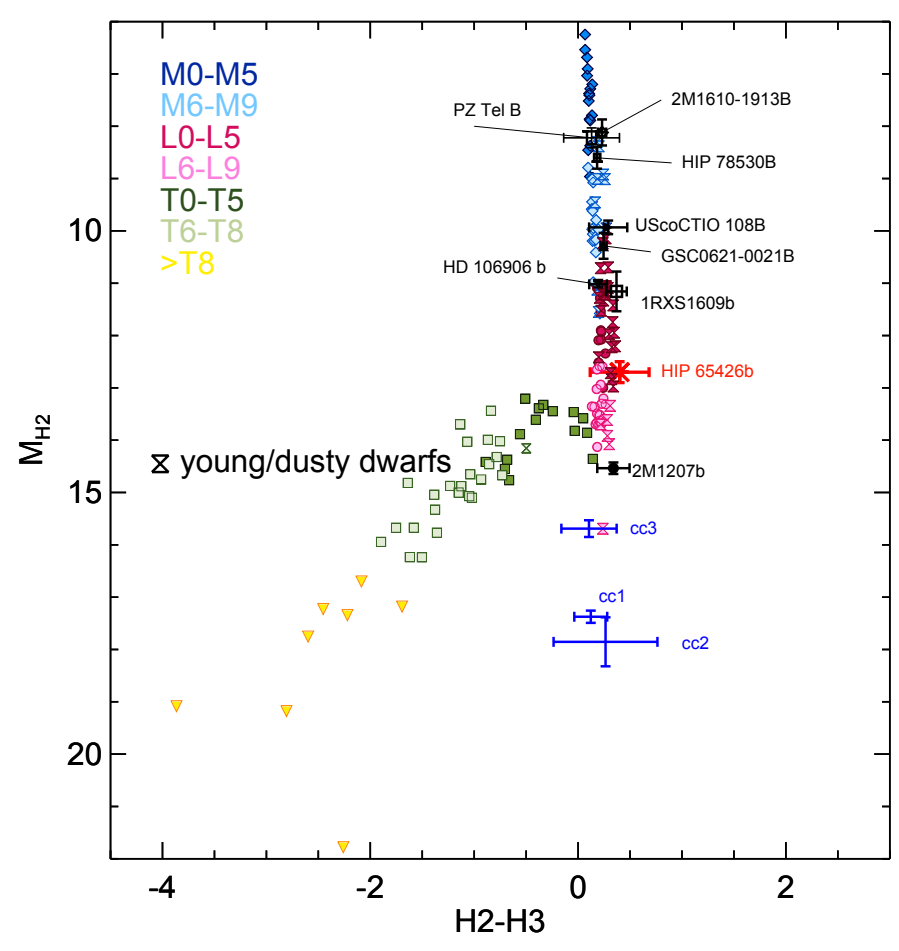

Fig. F.1. Color-magnitude diagram considering the SPHERE/IRDIS $\mathrm{H}_{2}$ and $\mathrm{H}_{3}$ photometry. HIP 65426 is indicated with error bars in red and the other companion candidates are shown in blue. 